\title{
Some New Perturbed Generalizations of Ostrowski- Grüss Type Inequalities for Bounded Differentiable Mappings and Applications
}

\author{
Shunfeng Wang ${ }^{1}$, Qiaoling Xue ${ }^{2}$ and Wenjun Liu ${ }^{2, *}$ \\ ${ }^{1}$ Binjiang College, Nanjing University of Information Science and Technology, Nanjing 210044, China \\ ${ }^{2}$ College of Mathematics and Statistics, Nanjing University of Information Science and Technology, Nanjing 210044, China
}

Received: 11 Feb. 2013, Revised: 13 Jun. 2013, Accepted: 16 Jun. 2013

Published online: 1 Sep. 2013

\begin{abstract}
In this note, we establish some new perturbed generalizations of Ostrowski-Grüss type inequalities with a parameter for bounded differentiable mappings. Our results in special cases give new bounds for Ostrowski-Grüss type or Ostrowski type inequalities. Some applications to probability density functions are also given.
\end{abstract}

Keywords: Ostrowski-Grüss type inequalities, Differentiable mappings, Ostrowski inequality

\section{Introduction}

The following Ostrowski-Grüss type integral inequality was proved by Dragomir and Wang in [2].

Theorem 1.Let $I \subset \mathbf{R}$ be an open interval, $a, b \in I, a<b$. If $f: I \rightarrow \mathbf{R}$ is a differentiable function such that there exist constants $\gamma, \Gamma \in \mathbf{R}$, with $\gamma \leq f^{\prime}(x) \leq \Gamma, x \in[a, b]$. Then for all $x \in[a, b]$, we have

$$
\begin{aligned}
& \left|f(x)-\frac{f(b)-f(a)}{b-a}\left(x-\frac{a+b}{2}\right)-\frac{1}{b-a} \int_{a}^{b} f(t) d t\right| \\
\leq & \frac{1}{4}(b-a)(\Gamma-\gamma) .
\end{aligned}
$$

In [1], Cheng not only gave a sharp version of the above inequality but also generalized it as follows.

Theorem 2.Let the assumptions of Theorem 1 hold. Then

$$
\begin{aligned}
& \left|\frac{1}{2} f(x)-\frac{(x-b) f(b)-(x-a) f(a)}{2(b-a)}-\frac{1}{b-a} \int_{a}^{b} f(t) d t\right| \\
\leq & \frac{(x-a)^{2}+(b-x)^{2}}{8(b-a)}(\Gamma-\gamma),
\end{aligned}
$$

for all $x \in[a, b]$.
In [9], two perturbations of an Ostrowski type inequality were established. Recently in [10], the present authors obtained the following perturbed generalization of Ostrowski-Grüss type inequality for bounded differentiable mapping with a parameter, which not only generalize Theorem 2, but also give some other interesting inequalities as special cases.

Theorem 3.Let the assumptions of Theorem 1 hold. Then

$$
\begin{aligned}
& \mid\left(1-\frac{\lambda}{2}\right) f(x)-\lambda \frac{(x-b) f(b)-(x-a) f(a)}{2(b-a)} \\
& -\frac{\Gamma+\gamma}{2}(1-\lambda)\left(x-\frac{a+b}{2}\right)-\frac{1}{b-a} \int_{a}^{b} f(t) d t \mid \\
& \leq\left(1-\lambda+\frac{\lambda^{2}}{2}\right) \frac{(x-a)^{2}+(b-x)^{2}}{4(b-a)}(\Gamma-\gamma),
\end{aligned}
$$

for all $x \in[a, b]$ and $\lambda \in[0,2]$.

More recently, Theorem 3 was proved for general time scales by Tuna and Daghan in [8]. Sarikaya [6] established a similar inequality of Ostrowski-type involving functions of two independent variables. In [7], Set, Sarikaya and Ahmad improved and further generalized some Čebyšev type inequalities involving functions whose derivatives belong to $L_{p}$ spaces via

\footnotetext{
*Corresponding author e-mail: wjliu@ nuist.edu.cn
} 
certain integral identities. In [3], Liu derived the following sharp generalized Ostrowski-Grüss inequality by using a variant of Grüss inequality (See also $[4,5]$ for other related works).

Theorem 4.Let the assumptions of Theorem 1 hold. Then

$$
\begin{aligned}
& \mid\left(1-\frac{\lambda}{2}\right) f(x)-\lambda \frac{(x-b) f(b)-(x-a) f(a)}{2(b-a)} \\
& \quad-S(1-\lambda)\left(x-\frac{a+b}{2}\right)-\frac{1}{b-a} \int_{a}^{b} f(t) d t \mid \\
& \leq \frac{\Gamma-\gamma}{2} I(\lambda, x),
\end{aligned}
$$

for all $x \in[a, b]$ and $\lambda \in[0,2]$, where $S=(f(b)-f(a)) /(b-a)$ and

$$
I(\lambda, x)=\left\{\begin{array}{l}
{\left[\frac{a+b}{2}-\left(1-\frac{\lambda}{2}\right) a-\frac{\lambda}{2} x\right]^{2},} \\
x \in\left[a, \frac{a+(1-\lambda) b}{2-\lambda}\right], \\
\frac{1}{4}\left[1+(\lambda-1)^{2}\right]\left[(x-a)^{2}+(b-x)^{2}\right], \\
x \in\left(\frac{a+(1-\lambda) b}{2-\lambda}, \frac{(1-\lambda) a+b}{2-\lambda}\right), \\
{\left[\frac{\lambda}{2} x+\left(1-\frac{\lambda}{2}\right) b-\frac{a+b}{2}\right]^{2},} \\
x \in\left[\frac{(1-\lambda) a+b}{2-\lambda}, b\right]
\end{array}\right.
$$

for $\lambda \in[0,1]$, and

$$
I(\lambda, x)=\left\{\begin{array}{c}
{\left[\frac{a+b}{2}-\frac{\lambda}{2} a-\left(1-\frac{\lambda}{2}\right) x\right]^{2},} \\
x \in\left[a, \frac{a+(\lambda-1) b}{\lambda}\right], \\
\frac{1}{4}\left[1+(\lambda-1)^{2}\right]\left[(x-a)^{2}+(b-x)^{2}\right], \\
x \in\left(\frac{a+(\lambda-1) b}{\lambda}, \frac{(\lambda-1) a+b}{\lambda}\right), \\
{\left[\left(1-\frac{\lambda}{2}\right) x+\frac{\lambda}{2} b-\frac{a+b}{2}\right]^{2}} \\
x \in\left[\frac{(\lambda-1) a+b}{\lambda}, b\right]
\end{array}\right.
$$

for $\lambda \in[1,2]$.

In this note, motivated by above research, we shall establish another perturbed generalization of Ostrowski-Grüss type inequalities with a parameter for bounded differentiable mappings. Our results in special cases give new bounds for Ostrowski-Grüss type or Ostrowski type inequalities. Some applications to probability density functions are also given.

\section{Main Results}

Theorem 5.Let $I \subset \mathbf{R}$ be an open interval, $a, b \in I, a<b$. If $f: I \rightarrow \mathbf{R}$ is a differentiable function such that there exist constants $\gamma, \Gamma \in \mathbf{R}$, with $\gamma \leq f^{\prime}(x) \leq \Gamma, x \in[a, b]$, then, we have

$$
\begin{aligned}
& \mid\left(1-\frac{\lambda}{2}\right) f(x)-\lambda \frac{(x-b) f(b)-(x-a) f(a)}{2(b-a)} \\
& -\gamma(1-\lambda)\left(x-\frac{a+b}{2}\right)-\frac{1}{b-a} \int_{a}^{b} f(t) d t \mid \\
\leq & \frac{1}{2}[1+|\lambda-1|]\left[\frac{b-a}{2}+\left|x-\frac{a+b}{2}\right|\right](S-\gamma),
\end{aligned}
$$

and

$$
\begin{aligned}
& \mid\left(1-\frac{\lambda}{2}\right) f(x)-\lambda \frac{(x-b) f(b)-(x-a) f(a)}{2(b-a)} \\
& -\Gamma(1-\lambda)\left(x-\frac{a+b}{2}\right)-\frac{1}{b-a} \int_{a}^{b} f(t) d t \mid \\
\leq & \frac{1}{2}[1+|\lambda-1|]\left[\frac{b-a}{2}+\left|x-\frac{a+b}{2}\right|\right](\Gamma-S),
\end{aligned}
$$

for all $x \in[a, b]$ and $\lambda \in[0,2]$, where $S=(f(b)-f(a)) /(b-a)$.

Proof.Let us define the following mapping as in [10]:

$K(x, t)=\left\{\begin{array}{l}t-\left(a+\lambda \frac{x-a}{2}\right), t \in[a, x], \\ t-\left(b-\lambda \frac{b-x}{2}\right), t \in(x, b] .\end{array}\right.$

Integrating by parts, we have

$$
\begin{aligned}
& \frac{1}{b-a} \int_{a}^{b} K(x, t) f^{\prime}(t) d t=\left(1-\frac{\lambda}{2}\right) f(x) \\
& -\lambda \frac{(x-b) f(b)-(x-a) f(a)}{2(b-a)}-\frac{1}{b-a} \int_{a}^{b} f(t) d t .
\end{aligned}
$$

We also have

$$
\frac{1}{b-a} \int_{a}^{b} K(x, t) d t=(1-\lambda)\left(x-\frac{a+b}{2}\right) .
$$

Let $C \in \mathbf{R}$ be a constant. From (10) and (11), it follows that

$$
\begin{aligned}
& \frac{1}{b-a} \int_{a}^{b} K(x, t)\left[f^{\prime}(t)-C\right] d t \\
= & \left(1-\frac{\lambda}{2}\right) f(x)-\lambda \frac{(x-b) f(b)-(x-a) f(a)}{2(b-a)} \\
& -C(1-\lambda)\left(x-\frac{a+b}{2}\right)-\frac{1}{b-a} \int_{a}^{b} f(t) d t .
\end{aligned}
$$

If we choose $C=\gamma$ in (12), then we get

$$
\begin{aligned}
& \frac{1}{b-a} \int_{a}^{b} K(x, t)\left[f^{\prime}(t)-\gamma\right] d t \\
= & \left(1-\frac{\lambda}{2}\right) f(x)-\lambda \frac{(x-b) f(b)-(x-a) f(a)}{2(b-a)} \\
& -\gamma(1-\lambda)\left(x-\frac{a+b}{2}\right)-\frac{1}{b-a} \int_{a}^{b} f(t) d t .
\end{aligned}
$$


On the other hand, we have

$$
\begin{aligned}
& \left|\frac{1}{b-a} \int_{a}^{b} K(x, t)\left[f^{\prime}(t)-\gamma\right] d t\right| \\
\leq & \max _{t \in[a, b]}|K(x, t)| \frac{1}{b-a} \int_{a}^{b}\left|f^{\prime}(t)-\gamma\right| d t .
\end{aligned}
$$

We also have

$$
\max _{t \in[a, b]}|K(x, t)|=\frac{1}{2}[1+|\lambda-1|]\left[\frac{b-a}{2}+\left|x-\frac{a+b}{2}\right|\right]
$$

and (see [9])

$$
\frac{1}{b-a} \int_{a}^{b}\left|f^{\prime}(t)-\gamma\right| d t=S-\gamma
$$

From (14)-(16), it follows that

$$
\begin{aligned}
& \left|\frac{1}{b-a} \int_{a}^{b} K(x, t)\left[f^{\prime}(t)-\gamma\right] d t\right| \\
\leq & \frac{1}{2}[1+|\lambda-1|]\left[\frac{b-a}{2}+\left|x-\frac{a+b}{2}\right|\right](S-\gamma) .
\end{aligned}
$$

From (13) and (17) we see that (7) holds.

If we choose $C=\Gamma$ in (12), then we get (8) similarly.

Corollary 1.Under the assumptions of Theorem 5 and with $\lambda=1$, we have

$$
\begin{aligned}
& \left|\frac{1}{2} f(x)-\frac{(x-b) f(b)-(x-a) f(a)}{2(b-a)}-\frac{1}{b-a} \int_{a}^{b} f(t) d t\right| \\
\leq & \frac{1}{2}\left[\frac{b-a}{2}+\left|x-\frac{a+b}{2}\right|\right](S-\gamma)
\end{aligned}
$$

and

$$
\begin{aligned}
& \left|\frac{1}{2} f(x)-\frac{(x-b) f(b)-(x-a) f(a)}{2(b-a)}-\frac{1}{b-a} \int_{a}^{b} f(t) d t\right| \\
\leq & \frac{1}{2}\left[\frac{b-a}{2}+\left|x-\frac{a+b}{2}\right|\right](\Gamma-S),
\end{aligned}
$$

for all $x \in[a, b]$.

Corollary 2.Under the assumptions of Theorem 5 and with $\lambda=0$, we have

$$
\begin{aligned}
& \left|f(x)-\gamma\left(x-\frac{a+b}{2}\right)-\frac{1}{b-a} \int_{a}^{b} f(t) d t\right| \\
& \leq\left[\frac{b-a}{2}+\left|x-\frac{a+b}{2}\right|\right](S-\gamma)
\end{aligned}
$$

and

$$
\begin{aligned}
& \left|f(x)-\Gamma\left(x-\frac{a+b}{2}\right)-\frac{1}{b-a} \int_{a}^{b} f(t) d t\right| \\
& \leq\left[\frac{b-a}{2}+\left|x-\frac{a+b}{2}\right|\right](\Gamma-S),
\end{aligned}
$$

for all $x \in[a, b]$.
Corollary 3.Under the assumptions of Theorem 5 and with $\lambda=2$, we have

$$
\begin{aligned}
& \mid \gamma\left(x-\frac{a+b}{2}\right)-\frac{(x-b) f(b)-(x-a) f(a)}{b-a} \\
& -\frac{1}{b-a} \int_{a}^{b} f(t) d t \mid \\
\leq & {\left[\frac{b-a}{2}+\left|x-\frac{a+b}{2}\right|\right](S-\gamma) }
\end{aligned}
$$

and

$$
\begin{aligned}
& \mid \Gamma\left(x-\frac{a+b}{2}\right)-\frac{(x-b) f(b)-(x-a) f(a)}{b-a} \\
& -\frac{1}{b-a} \int_{a}^{b} f(t) d t \mid \\
& \leq\left[\frac{b-a}{2}+\left|x-\frac{a+b}{2}\right|\right](\Gamma-S),
\end{aligned}
$$

for all $x \in[a, b]$.

Corollary 4.Under the assumptions of Theorem 5 and with $x=\frac{a+b}{2}$, we have

$$
\begin{aligned}
& \mid\left(1-\frac{\lambda}{2}\right) f\left(\frac{a+b}{2}\right)+\frac{\lambda}{2} \frac{f(a)+f(b)}{2} \\
& -\frac{1}{b-a} \int_{a}^{b} f(t) d t \mid \leq \frac{b-a}{4}[1+|\lambda-1|](S-\gamma)
\end{aligned}
$$

and

$$
\begin{aligned}
& \mid\left(1-\frac{\lambda}{2}\right) f\left(\frac{a+b}{2}\right)+\frac{\lambda}{2} \frac{f(a)+f(b)}{2} \\
& -\frac{1}{b-a} \int_{a}^{b} f(t) d t \mid \leq \frac{b-a}{4}[1+|\lambda-1|](\Gamma-S),
\end{aligned}
$$

for all $\lambda \in[0,2]$.

Corollary 5.Under the assumptions of Theorem 5 and with $x=a$, we have

$$
\begin{aligned}
& \mid\left(1-\frac{\lambda}{2}\right) f(a)+\frac{\lambda}{2} f(b)+\frac{\gamma}{2}(1-\lambda)(b-a) \\
& -\frac{1}{b-a} \int_{a}^{b} f(t) d t \mid \leq \frac{b-a}{2}[1+|\lambda-1|](S-\gamma)
\end{aligned}
$$

and

$$
\begin{aligned}
& \mid\left(1-\frac{\lambda}{2}\right) f(a)+\frac{\lambda}{2} f(b)+\frac{\Gamma}{2}(1-\lambda)(b-a) \\
& -\frac{1}{b-a} \int_{a}^{b} f(t) d t \mid \leq \frac{b-a}{2}[1+|\lambda-1|](\Gamma-S),
\end{aligned}
$$

for all $\lambda \in[0,2]$. 
Corollary 6.Under the assumptions of Theorem 5 and with $x=b$, we have

$$
\begin{aligned}
& \mid\left(1-\frac{\lambda}{2}\right) f(b)+\frac{\lambda}{2} f(a)-\frac{\gamma}{2}(1-\lambda)(b-a) \\
& -\frac{1}{b-a} \int_{a}^{b} f(t) d t \mid \leq \frac{b-a}{2}[1+|\lambda-1|](S-\gamma)
\end{aligned}
$$

and

$$
\begin{aligned}
& \mid\left(1-\frac{\lambda}{2}\right) f(b)+\frac{\lambda}{2} f(a)-\frac{\Gamma}{2}(1-\lambda)(b-a) \\
& -\frac{1}{b-a} \int_{a}^{b} f(t) d t \mid \leq \frac{b-a}{2}[1+|\lambda-1|](\Gamma-S),
\end{aligned}
$$

for all $\lambda \in[0,2]$.

Remark. We point out that, as in [8] and [6], Theorem 5 may be proved for general time scales or be similarly extended to inequalities involving functions of two independent variables. The details are left for the interested reader.

\section{Application to probability density functions}

Now, let $X$ be a random variable taking values in the finite interval $[a, b]$, with the probability density function $f:[a, b] \rightarrow \mathbf{R}^{+}$and with the cumulative distribution function

$$
F(x)=\operatorname{Pr}(X \leq x)=\int_{a}^{x} f(t) d t .
$$

The following result holds:

Theorem 6. With the above assumptions and that the probability density function $f$ satisfies $\gamma \leq f(x) \leq \Gamma$, $x \in[a, b]$ for some constants $\gamma, \Gamma \in \mathbf{R}^{+}$, then, we have

$$
\begin{aligned}
& \mid\left(1-\frac{\lambda}{2}\right) \operatorname{Pr}(X \leq x)-\frac{\lambda}{2} \frac{x-b}{b-a} \\
& -\gamma(1-\lambda)\left(x-\frac{a+b}{2}\right)-\frac{b-E(X)}{b-a} \mid \\
\leq & \frac{1}{2}[1+|\lambda-1|]\left[\frac{b-a}{2}+\left|x-\frac{a+b}{2}\right|\right]\left(\frac{1}{b-a}-\gamma\right),
\end{aligned}
$$

and

$$
\begin{aligned}
& \mid\left(1-\frac{\lambda}{2}\right) \operatorname{Pr}(X \leq x)-\frac{\lambda}{2} \frac{x-b}{b-a} \\
& -\Gamma(1-\lambda)\left(x-\frac{a+b}{2}\right)-\frac{b-E(X)}{b-a} \mid \\
\leq & \frac{1}{2}[1+|\lambda-1|]\left[\frac{b-a}{2}+\left|x-\frac{a+b}{2}\right|\right]\left(\Gamma-\frac{1}{b-a}\right),
\end{aligned}
$$

for all $x \in[a, b]$ and $\lambda \in[0,2]$, where $E(X)$ is the expectation of $X$.
Proof.By choosing $f=F$ in (7) and (8), and taking into account $F(a)=0, F(b)=1$ and

$$
E(X)=\int_{a}^{b} t d F(t)=b-\int_{a}^{b} F(t) d t
$$

we obtain (30) and (31).

In particular, we have:

Corollary 7. With the above assumptions, we have the inequalities

$$
\begin{aligned}
& \left|\frac{1}{2} \operatorname{Pr}(X \leq x)-\frac{1}{2} \frac{x-b}{b-a}-\frac{b-E(X)}{b-a}\right| \\
\leq & \frac{1}{2}\left[\frac{b-a}{2}+\left|x-\frac{a+b}{2}\right|\right]\left(\frac{1}{b-a}-\gamma\right),
\end{aligned}
$$

and

$$
\begin{aligned}
& \left|\frac{1}{2} \operatorname{Pr}(X \leq x)-\frac{1}{2} \frac{x-b}{b-a}-\frac{b-E(X)}{b-a}\right| \\
\leq & \frac{1}{2}\left[\frac{b-a}{2}+\left|x-\frac{a+b}{2}\right|\right]\left(\Gamma-\frac{1}{b-a}\right),
\end{aligned}
$$

for all $x \in[a, b]$.

Proof. We set $\lambda=1$ in Theorem 6 .

Corollary 8. With the above assumptions, we have the inequalities

$$
\begin{aligned}
& \left|\gamma\left(x-\frac{a+b}{2}\right)-\frac{x-b}{b-a}-\frac{b-E(X)}{b-a}\right| \\
\leq & {\left[\frac{b-a}{2}+\left|x-\frac{a+b}{2}\right|\right]\left(\frac{1}{b-a}-\gamma\right), }
\end{aligned}
$$

and

$$
\begin{aligned}
& \left|\Gamma\left(x-\frac{a+b}{2}\right)-\frac{x-b}{b-a}-\frac{b-E(X)}{b-a}\right| \\
\leq & {\left[\frac{b-a}{2}+\left|x-\frac{a+b}{2}\right|\right]\left(\Gamma-\frac{1}{b-a}\right), }
\end{aligned}
$$

for all $x \in[a, b]$.

Proof. We set $\lambda=2$ in Theorem 6.

\section{Acknowledgement}

The authors are grateful to the anonymous referees for a careful checking of the details and for helpful comments that improved this paper. 


\section{References}

[1] X. L. Cheng, Improvement of some Ostrowski-Grüss type inequalities, Comput. Math. Appl., 42, 109-114 (2001).

[2] S. S. Dragomir and S. Wang, An inequality of OstrowskiGrüss type and its applications to the estimation of error bounds for some special means and for some numerical quadrature rules, Comput. Math. Appl., 33, 15-20 (1997).

[3] Z. Liu, A sharp generalized Ostrowski-Grüss inequality, Tamsui Oxf. J. Math. Sci., 24, 175-184 (2008).

[4] W. J. Liu and J. Park, A generalization of the companion of Ostrowski-like inequality and applications, Applied Mathematics \& Information Sciences, 7, 273-278 (2013).

[5] M. Matić, J. Pečarić and N. Ujević, Improvement and further generalization of inequalities of Ostrowski-Grüss type, Comput. Math. Appl., 39, 161-175 (2000).

[6] M. Z. Sarikaya, On the Ostrowski type integral inequality, Acta Math. Univ. Comenian. (N.S.,) 79, 129-134 (2010).

[7] E. Set, M. Z. Sarikaya, and F. Ahmad, A generalization of Čebyšev type inequalities for first differentiable mappings, Miskolc Mathematical Notes, 12, 245-253 (2011).

[8] A. Tuna and D. Daghan, Generalization of Ostrowski and Ostrowski-Grüss type inequalities on time scales, Comput. Math. Appl., 60, 803-811 (2010).

[9] N. Ujević, Perturbations of an Ostrowski type inequality and applications, Int. J. Math. Math. Sci., 32, 491-500 (2002).

[10] S. F. Wang, Q. L. Xue and W. J. Liu, Further generalization of Ostrowski-Grüss type inequalities, Advances in Applied Mathematical Analysis, 3, 17-20 (2008). 